\title{
A Face Recognition System using Directional Binary Code Algorithm and Multi-SVM
}

\author{
M. Tamilselvi, S. Karthikeyan
}

\begin{abstract}
Over past few years, face recognition technology plays an important function in the development of biometric identifier with less time consuming and computational overhead. Many researchers were put their effort to develop face recognition algorithm involves three distinct steps such as detection, unique faceprint creation and finally verification. Traditional Local binary pattern based face recognition system slow down the recognition speed, high computational complexity and does not give the directional data of the picture. In order to overcome the above limitation, a novel face recognition system is proposed by employing the advantage of Directional Binary Code (DBC) feature extraction method. The face images features are extracted from DBC are generally smoother than other feature extraction methods. The images with blur creation, pose changes, and illumination is applied and stored in the database. For blur creation various filters such as Average filter, Gaussian filter and Motion filter are used. By using Directional Binary Code method, the face is detected and extracted. Then the same algorithm is used for input images and with help of Multi-SVM classifier multiple images in the database is compared and shows the matched images. Finally, simulation result shows the implemented results in term of its recognition speed and computation complexity.
\end{abstract}

Index Terms:- Directional Binary Code; Blur creation; MultiSVM; Face recognition; Gaussian Filter; Local Binary Pattern.

\section{INTRODUCTION}

Face recognition is a computer based development which finds the differences among the collected and stored human pictures in the database. It is in like manner implying the psychological strategy by which individuals find and deal with faces in a visual scene. Face acknowledgment estimation base on the recognizable proof of the frontal human faces. It is like picture recognizable proof in which the image of the individual is composed a small piece at once. Picture matches with picture set away in the database. Any facial part changes in the database will refute the planning method. Immediately, the possible human eye zones are perceived by testing all the valley areas in the diminish measurement picture. By then the genetic computation is used to create all the possible face regions which fuse the eyebrows, the iris, the nostril and the mouth corners. Each possible face contender is institutionalized to reduce lightning effect caused in view of uneven illumination and the shirring sway due to head advancement. The agility estimation of every hopeful is calculated depends on its prediction on the Eigen-faces. After different sequences, all the face candidates with a high ruggedness regard are picked for further affirmation. At this particular point, the face uniformity is evaluated and the nearness of the typical facial features are established on every face contender.

This paper is composed as pursues: Present area gives the short presentation on the procedures of detecting face and the significance of the suggested face recognition framework. Section II shows the related work done on this face recognition system in past few years. Section III describes about the traditional face recognition system using local binary pattern. Section IV explains the proposed face recognition system and its advantages. Section $\mathrm{V}$ analysis the recognition system in terms of speed and computational complexity using MATLAB software. Finally concludes the paper.

\section{Authors Contribution:}

A Face Recognition is the most challenges in many fields such as Biomedical, Agriculture and also in various authentication purposes [1]. The primary point of this work is on improving the effective image retrieval efficiency. The image execution recovery relies upon the element extraction. The proposed algorithm is Directional Binary Code where the center pixel is compared with the neighbor pixel by certain directions. Image Blurring is done to retrieve the best matches of the image. The methods for blurring an image can be done using low pass filter such as Average filter, Gaussian filter and Motion filter whereas convolution display for space invariant blur method only detect the edge not the out plane movement and in plane turn. [2] Also it cannot detect the edge of an image when the image gets shake. Therefore, in this paper motion filter is used to overcome the drawback.

\section{II.RELATED WORKS}

This section was carried out in three different stages via to identify the cause of the problem, to find the optimal solution to the problem without affecting the production unit and to standardize and regularize the process in the organization as well as to establish the knowledge base as a part of the production within the organization

Local Binary Pattern (LBP) is generally embraced on effective image feature depiction with straightforwardness. 
A simple and systematic broadly embraced method for picture feature interpretation is Local binary pattern (LBP)[2]. To portray the shading pictures, consolidation of Local binary patterns of all channel is needed. The customary method for binary combination is to just link the Local binary patterns of every channel, yet it expands the size of the example. So as to adapt to this issue, Abhijith[2] introduces a innovative approach to depict a image for LBPs received from various channels, it is necessary to implement the two pattern based adder and decoder in order to mix the local binary patterns from more than single channel. Retrieving test of images are done to recognize the acceptability of the suggested methodologies along with the analysis of existing methods for more than a single channel strategy. The samples are efficiently working till twelve standard normal scene and saturation structure picture databases, for example, Corel-1k, MIT-VisTex, USPTex, Colored Brodatz, etc. It is seen that the presented multichannel adder and decoder based local binary patterns fundamentally enhances the recovery execution on every database and beats other multichannel format methodologies as far as the normal recovery accuracy and normal recovery rate.

Throughout late decades, different face recognition systems have been proposed in PC vision, and most of them use thorough face images for individual identification. Under certain practical situations specifically on some unconfined situations, face images are obstructed by various entities, and impossible to acquire completely allencompassing human images for identification. So as to determine this, RenliangWeng suggested an another limited method where theexcitement of individuals due to partial emergence of their faces were perceived. Suggested method, took pair of display image and analyze face fix. It at first distinguished the key points and concentrates on the surrounding consistency peak properties. In that moment, a hearty point

At that point, coordination of hearty point setting up is utilized to discriminatively coordinate these two extricated nearby capabilities, where both the textural information and geometrical information of neighborhood features are explicitly used for planning at the same time. Finally, the resemblance of two images are changed over as the detachment between these two balanced capacities. Preliminary outcomes on four open image enlightening files exhibit the sufficiency of the suggested methodology.

Majeed introduces a technique for image deblurring and remaking of HDR images utilizing transformation spread functions(TSFs), which is legitimately evaluated from locally derived point spread functions (PSFs) by accomplishing their relationship. It likewise figuring the quality Measurement parameters of pictures [4].

Shabat suggested a gradient-based adaptive interpolation based super-resolution technique in which the original image local gradient can be taken by interpolation coefficients including with the consideration of the separation between the interpolated pixel and neighboring valid pixel. The littler the neighborhood angle of a pixel is, the more impact it ought to have on the inserted pixel. The small local gradient of a pixel having more influence on interpolated pixel and at the last the high resoluted image is deblurred with the help of wiener filter. The test results shows improvement in enhancement of edges, restoring image quality and on its robustness.

ZhenhuaGuo introduces a technique where a face can be recognized with only one training image per subject even under inconsistent illumination conditions. This strategy depends on the ongoing outcome which exhibited that the arrangement of pictures of a curved Lambert Ian object acquired over a broad assortment of various light situations which are estimated precisely over low-dimensional linear subspace. Proposed framework recovers premise images spreading over this space from only one picture taken under subjective enlightenment conditions. To start with, utilizing a bootstrap set comprising of 3D face models, it processes a factual model for every basis image. When preparing, given a novel face image under self-assertive illumination, it recovers a lot of images for this face which are having the maximum probability. At the time of assessment, the face can be recognized which is having weighted combination of basis images closest to the face image which is under test. This method of continuous testing will tend to improve the rate of recognition over various range of lighting conditions there by helps to improve the level of accuracy.

Here not much concentration is given on linear kernel along with SVM classifier which is run on Parallel Processor Array. The fundamental target of their pursuit has been to build up a unique usage of the SVM classifier on a Massively Parallel Processor Array stage while the compositional congestions of the hyper spectral picture classifiers were examined. Trying different things with medicinal pictures, the proportionate of the Single vector machine characterization had been utilized with three methodologies: i) single-and multi-core processing, ii) single-and multi-cluster analysis and iii) single-and double buffer execution. As needs be, a typical center preparing speedup of 11.8 has been cultivated while parallelizing the SVM gathering process in a single cluster. Unexpectedly, all-out speedup is upper-limited to 2.9 due to information correspondence represents $34.7 \%$ of the all-out execution time in the successive case. Utilizing a two-buffer philosophy, a complete speedup of 2.84 has been accomplished on a single cluster. Finally, achievable versatile rendition of direct SVM was illustrated. [18]

MrtyunjayGhosh [17] considers the basic normal sifting calculation in the field of picture handling. A very normal average filtering algorithm is suggested by MrtyunjayGhosh in the developing area of processing the picture which has been used to enhance the image. The proposed algorithm shows better utilization of cache which in turn reduces the cache misses when compared to previous one.

Gonzale [16] introduces a technique which is based on rank order filter used in the time varying image noise suppression. This type of temporal filter protects the image features while in motion and not in motion in an effective manner. Both spatial MMF and temporal median filters shown improvement in increasing image resolution and also in suppressing noise.

Published By:

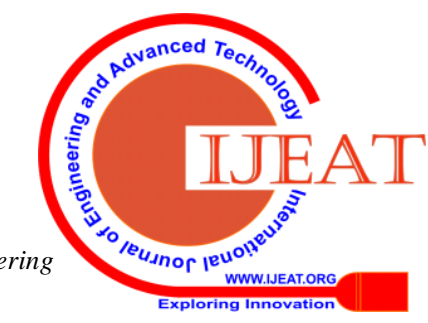


This work introduces new methodologies for motioncompensated spatio -temporal filters connected to advanced picture arrangements. A camera and object displacements are causing motion which is folded by the pixel intensities temporal correlation information for images which is varying with time. The temporal filters which can also be defined as Motion-compensated filters are applied in the path of motion which is assumed. In general, this paper says that the movement-compensated temporal filtering process can be considered as the product of double different operators where the primary one is a dependent factor of both movement-based facets partitions, and restricted affine modeling of regions in movement and the second one is used for correlation measurement of intensities image-byimage with the assumption of the path of the motion. In order to achieve the maximum results, in the path of motion the multiresolution filters are continuously applied and these can be used for spatial-temporal prediction, interpolation and smoothing. Here in this work [15], applications were given to the field of image succession coding and interpolation.

Santhosh [14] introduces a method to retrieve images and for face recognition applications is local directional mask maximum edge pattern. Local binary pattern (LBP) and the alternatives of LBP gather the connection among the middle pixel and its encompassing neighbors in an image which in turn cannot provide the stability for the differences caused by noise.

Though the proposed technique gathers the most extreme edge designs (MEP) and greatest edge pose designs (MEPP) from the greatness directional edges of image/picture. Instead of collecting the correlation among the mid pixel and to the neighboring pixels (LBP), the new technique introduced gathers the highest edge patterns(MEP) and highest edge pose patterns (MEPP) which can be derived by the face / image masked directional magnitude edges.

Depends upon the magnitude of directional edges (DE) and the pose of highest DE, the MEP and MEPP were coded after computing DE. In this regard, the stability of the introduced technique can be improvised by combining Multiresolution Gaussian filters along with it. In order to prove the improved performance of this technique with LBP and LBP variants, the analysis can be done in four different ways conducted on the data bases like open access series of imaging studies-magnetic resonance imaging, Brodatz, MIT VisTex and Extended Yale B for significant applications in retrieving of biomedical images, retrieval of texture and also in recognizing faces.

In the current trend, there is a huge need for common technique which can be used to retrieve and analyze the content based image since it is a major demand in facial recognition application in computer vision. In this context, a new technique is introduced where supporting vector machines are used for recognition of facial expressions.

In this model, 2D Gabor filters are implemented to extract the features first and then these features are trained to form a robust model. Generally, in six different ways the key vectors are handled and checked here. For six common facial expressions, the introduced method produces a great accuracy $(83.3 \%)$ and also the implementation becomes easy since the number of support vectors are reduced. Hence this method has more significance than other methods.

The detection of object, identification and recognizing the object is getting deteriorated while visual perception is damaged in ambiguous visual environments. Obscured pictures may likewise instigate negative full of feeling reactions to the visual condition, for example, dread or uncanniness. Nonetheless, the impact of picture obscure on the blur image is as yet open. The present examination researched the impacts of image obscure on detecting the object and also in the perception of uncanniness.

In an investigation, it displayed the photos of common images delineating an individual, an animal, or an object and requested that members to demonstrate whether the image portrayed a face or an animal and to rate how unequivocally they felt the image was uncanny. At different cut off frequencies of low pass filter, the images were blurred. It establishes that the more hazy images, specifically images filtered by the lower cutoff frequencies, were increasingly troublesome in face/creature recognition just as they were appraised as progressively uncanny $[11,12]$

\section{TRADITIONAL METHOD}

In current framework, picture ordering and retrieving is requesting increasingly much consideration because of its fast development in numerous spots. Picture recovery had a few applications, for example, in object recognition, biomedical, agriculture, and so forth.

The point of Content Based Image Retrieval (CBIR) is to separate the comparable pictures from the image given from big databases with the coordination of inquiry image with the images of the database. Coordinating of several pictures are encouraged with the coordination of real element descriptors (for example picture marks). It implies the execution of any picture recovery framework vigorously relies on the picture highlight descriptors being coordinated. Two images can be matched by coordinating their feature descriptors which is nothing but image signatures like color, texture, shape, gradient etc. For image feature descriptor, local pattern descriptors which are simple and effective in nature are used since.

In addition to the local binary pattern(LBP), other types of LBP also introduced for gray images which are characterized by single channel whereas multiple channels are used for characterizing the real time natural images as mentioned in fig.1. The traditional face recognition system having its limitations like

The length of the histogram will reduce the recognizing speed for huge data base.

- Since the center pixel is not taken into account, may miss the local structure over some specific situations.

- Complexity can be increased with the increase in number of features.

- The variance of the intensity values within region is very low.

- This method not providing the actual information of the image. 
- It is not robust on "flat" image areas since it is based on intensity differences.

In Euclidean distance approach it assumes that the sample points are distributed about the sample mean in a spherical manner

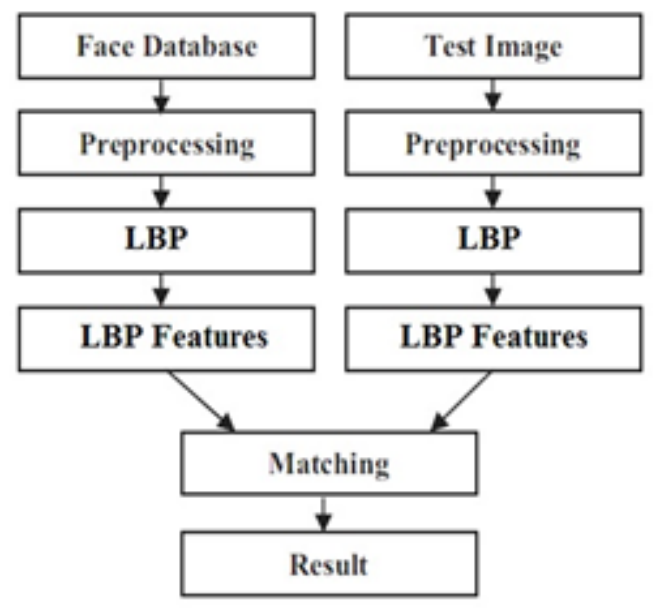

Fig. 1 Traditional Face Recognition system

\section{III.PROPOSED FACE RECOGNITION SYSTEM USING DIRECTIONAL BINARY CODE}

In order to overcome the above limitation, directional binary code based face recognition system is proposed instead of Local Binary Pattern. The images with blur creation, pose changes, and illumination is applied and stored in the database. For blur creation various filters such as Average filter, Gaussian filter and Motion filter are used. By using Directional Binary Code algorithm, the face is detected and extracted. Then the same algorithm is used for input images and with help of Multi-SVM classifier multiple images in the database is compared and shows the matched images.

A Face Recognition is the most challenges in many fields such as Biomedical, Agriculture and also in various authentication purposes. The primary purpose of this paper is to enhance the efficiency of the image retrieval by its performance. The retrieval efficiency of image can be evaluated based on the extraction of features. The proposed algorithm is Directional Binary Code where the center pixel is compared with the neighbor pixel by certain directions as shown in Fig.2. Image Blurring is done to retrieve the best matches of the image.

The methods for blurring an image can be done using low pass filter such as Average filter, Gaussian filter and Motion filter whereas convolution model for space invariant blur method only detect the edge not the out plane movement and in plane rotation. Also it cannot detect the edge of an image when the image gets shake. Therefore, motion filter is used to overcome the drawback. Proposed system consists of three modules such as Blur Creation, Database creation, DBC and matching.

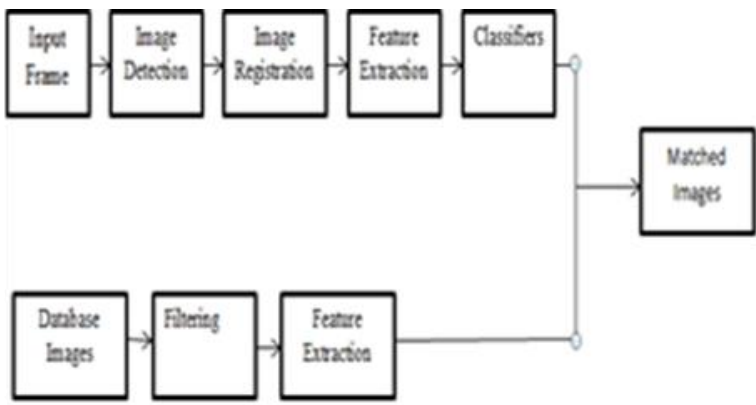

Fig.2 Proposed Face Recognition System

\subsection{Blur Creation}

Blurring is a kind of smoothening process which makes the sample image in different variations by using filters.

\section{Gaussian Blur}

Gaussian blur is also known as Gaussian smoothening. The image of an individual person is captured and stores it in file. After the cropping of original image, smoothening is completed by using Gaussian function. It reduces the noise and irrelevant details in an image. It is low pass filter. The properties of Gaussian filters are no sharp edges and no ringing effect. Gaussian blur effect is generated convolving image with obtained Gaussian value as shown in Fig.3.

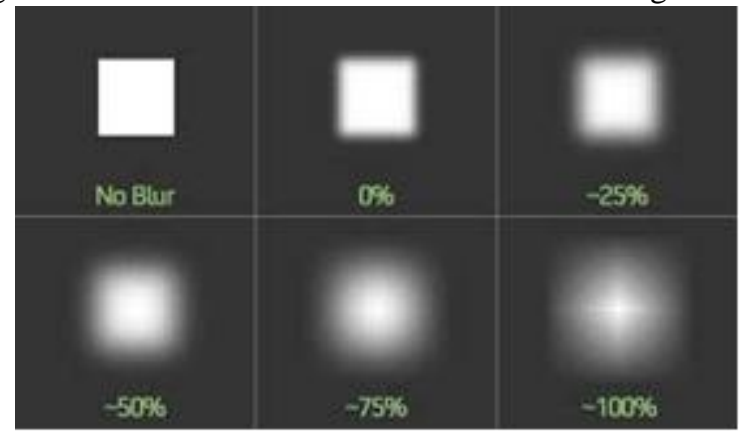

Fig. 3 Gaussian Blur

\section{Motion Blur}

It is the detectable attribute of continuously running object in a still image. Sometimes images are looking as blurred due to the moment of camera or unstable background. The images stored in database may appear in different blur shapes that increase the ability to find or compare the sample images with database images. Intensities are folded by motions which provide correlation information about the image. By applying motion for images that increases the number of sample copies for each original image. After creation of all copies, the copied images are stored in database. Noise suppression of time varying imagery is proposed by using temporal filter. It provides structure or shape for an image under motion and it does not require motion compensation preprocessing

\section{Average blur}


Blur is created for the image by setting each pixel equal to the average pixel value of its specified box neighborhood. Average filters also known as box filter or average filter. It uses advance version of average filtering algorithm in image processing and it creates better usage of resource than original average filtering. The order of the filter must be in odd. The sum of all elements inside the image must be equal to one so that all elements should be same. The blur can be increased by increasing the size of the mask.
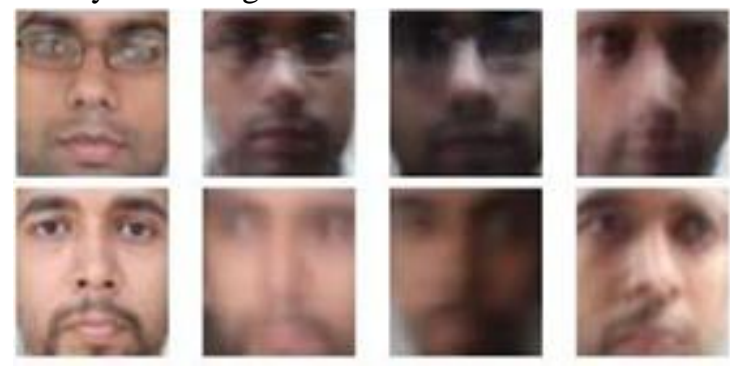

Fig. 3 Processed images

\subsection{Database Creation}

Here various set of images of different persons are obtained from camera with variations in pose and illumination and stored to use it as a data base.

\subsection{DBC and Matching}

Normally face recognition is based upon identification of feature vector from face. So that only comparison of sample and database images quiet easy to do. Feature extraction can be easily done by using directional binary code. DBC compares any pixel in an image along with some direction (0 $\square, 40 \square, 90,135 \square$ degree). By using DBC, spatial information about the pixel determined easily than LBP ashown in Fig. 4 and Fig.5.

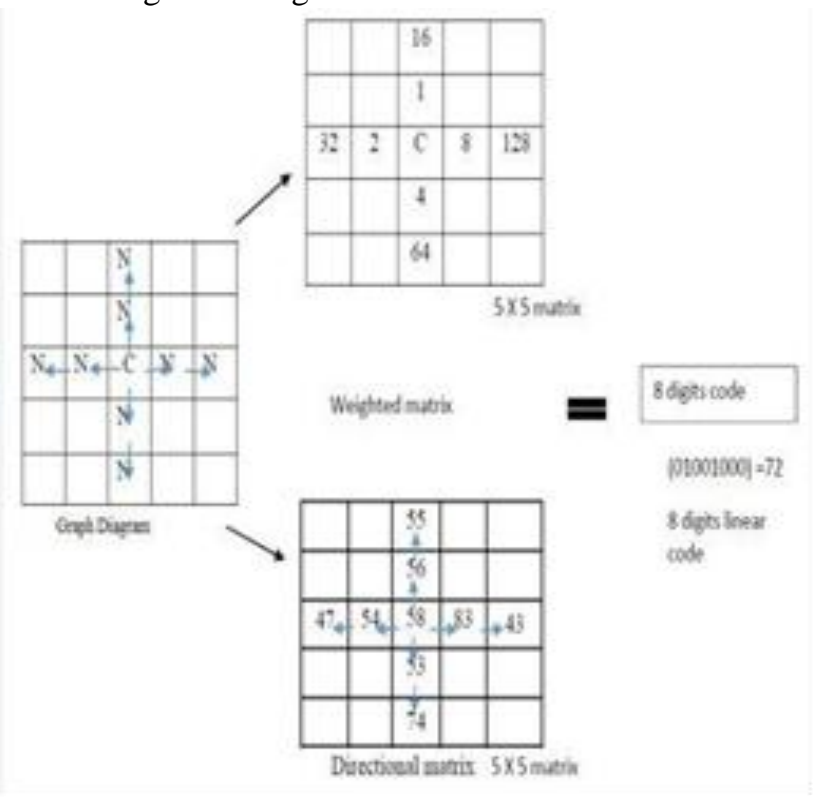

Fig. 4 DBC basic operator by $0 \square$ and $90 \square$

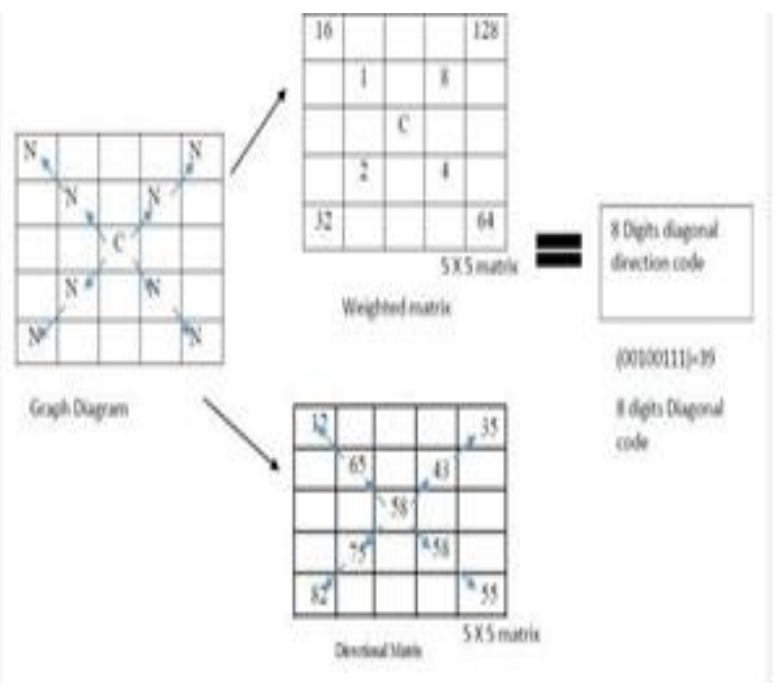

Fig.5 DBC basic operator by $45 \square$ and $135 \square$

\section{DBC algorithm}

The directional edge information of an image is encoded by applying DBC which catches the spatial correlation among any two of neighboring pixels within a particular section in a particular angle which is same as normal key factor. It retrieves many spatial related things than LBP. Let $\mathrm{Zi}, \mathrm{j}$ be a point in a cell, four directional derivatives at $\mathrm{Zi}, \mathrm{j}$ are given

$$
\begin{aligned}
& \mathrm{d}=1, \\
& I^{`} 0, d(Z i, j)=I(Z i, j)-I(Z i, j-d) ; \\
& I^{`} 45, d(Z i, j)=I(Z i, j)-I(z i-d, j+d) ; \\
& I^{\prime} 90, d(Z i, j)=I(Z i, j)-I(z i-d, j) ; \\
& I^{\prime} 135, d(Z i, j)=I(Z i, j)-I(z i-d, j-d) \text {; }
\end{aligned}
$$

The dimension of the new image of size $50 * 50$ is sectioned into 100 cells of $5 * 5$ matrixes. Table lindicates a $3 * 3$ nearby center on I $\mathrm{Zi}, \mathrm{j}$ which has been taken out of $5 * 5$ cell size, where each cell contributing one coefficient.

\section{Matching}

Classifier used as a function in the implementation of classification algorithm. Classifier maps input data. Classifier places a major role in speech recognition, video tracking, pattern recognition, biometric identification and handwriting recognition. The proposed method is MultiSVM classifier. The classification with more than two classes is known as Multi-SVM. The process is as follows: 1.Builds classifier for each class where the group of input image stored under a single file. 2. For each sample image, classifier applied separately. 3. By using classifier, it provides a value for each pixel in an image.4. Depending upon the values, sample image compared with database image. 5 . The performance of classifier determined by using confusion matrix as shown in Fig. 6. 
- Given a new point $\mathbf{x}$, we can score its projection onto the hyperplane normal:

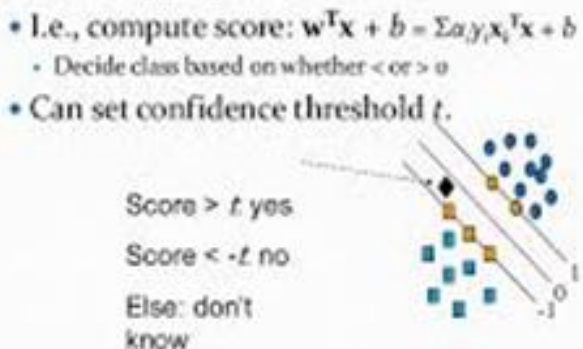

knON

Fig.6 Multi SVM classifier

\section{IV.RESULTS AND DISCUSSION}

Face is detected by camera through the viola jones algorithm inbuilt in the MATLAB software. Through directional binary code the feature is extracted and the pixels in the picture are calculated.

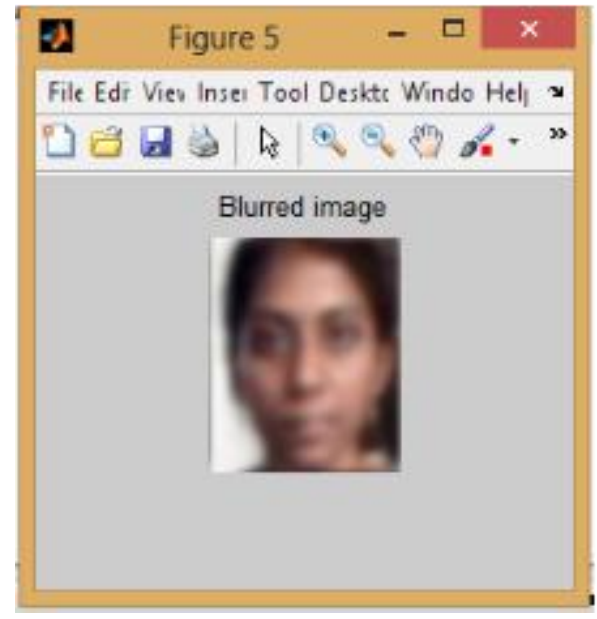

Fig. 6 (a) Blurred image

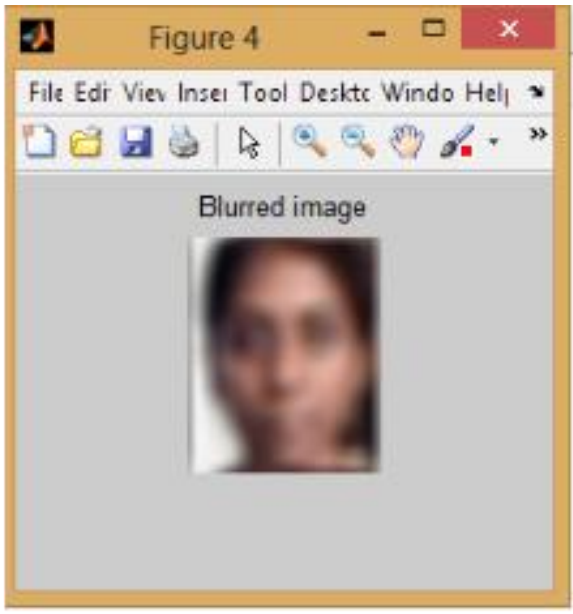

Fig.6 (b)Blurred image

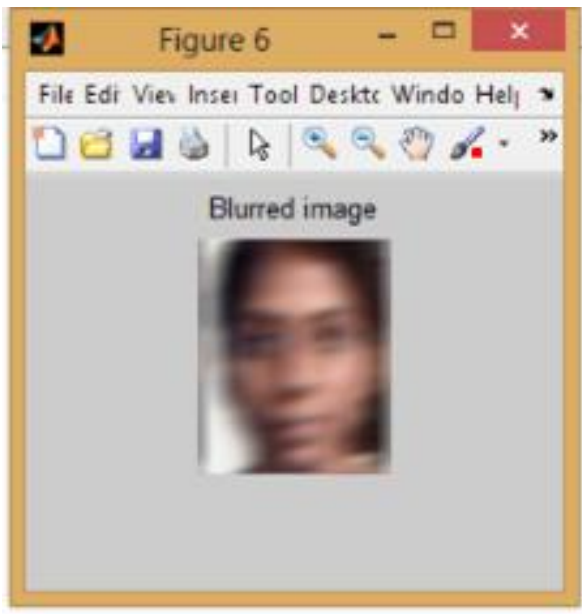

Fig. 6 ( c ) Blurred image

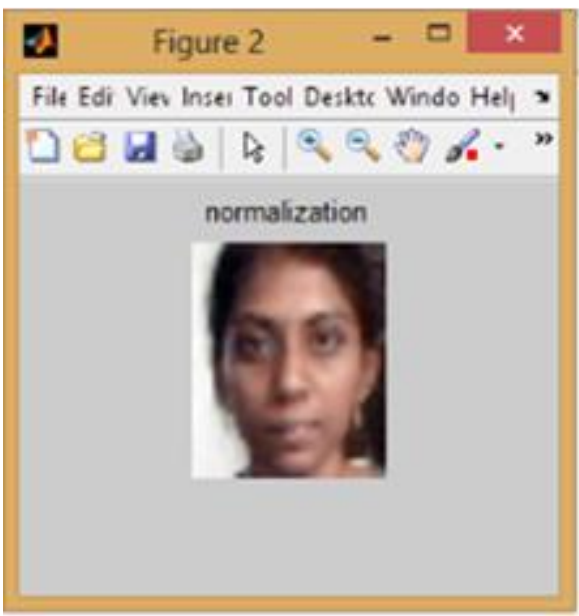

Fig. 7 Normalization

This experiment has been conducted on the ORL database which contains 400 images from 40 individuals in that 10 of each person with several luminance, expression and pose conditions. It'sgrayscale image and the resolution is $112 \times$ 92. Error rate is measured by applying various classifier. It's obvious that the performance of SVM classifier is efficient when compared withBP neural network and Nearest center criterion.

\begin{tabular}{|} 
Table 1: Comparison of Error Rates \\
\begin{tabular}{|c|c|}
\hline Classifier & Error Rate \\
\hline BPNN & $10 \%$ \\
\hline NCC & $7.5 \%$ \\
\hline Proposed Classifier & $4 \%$ \\
\hline
\end{tabular}
\end{tabular}

Multi class SVM was demonstrated based on the FERET database where more than 400 frontal images were stored. In order to facilitate a trading mark for the better performance of the algorithm, PCA based algorithm on the same set of images were given to compare the performance.

The Principal Component Analysis algorithm founds facets over L2 nearest neighbor classifier. At the same time the radial based kernel was used to mention the SVM-based algorithms. The recognition rate of PCA is about 54\% and of SVM is in between $77-78 \%$ which shows the effective usage of facet space when compared to the basic analysis in Principal Component Analysis algorithm.

Published By: Blue Eyes Intelligence Engineering

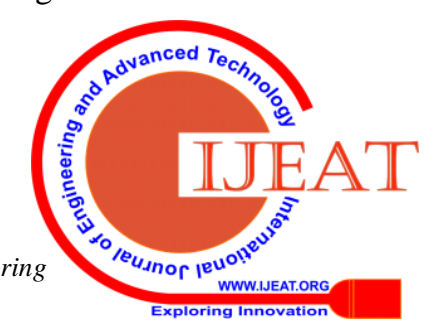


We implemented our proposed method in Georgiatech face database where the 5 images are selected in random manner in order to create the training set and also to test in different manner. From each person 10 different pictures are taken so that the data set consists of 50 images for experimental testing. The images stored in the training set are differ from each other by various factors like position change, expression change and wearing some thing or not like etc. The face direction is varied in left and right by 0-25 degrees. These kind of images which have been stored for training and testing purpose are preprocessed to achieve the recognition rate of about $97 \%$.

This implementation of the suggested technique in CVL face dataset, which consists of face images of 114 persons with seven images per person. Six samples are collected from the database for further evaluation. It is compared with SIFT technique.

Table 2: Comparison of Recognition Rate

\begin{tabular}{|l|l|l|}
\hline Training Data & SIFT & $\begin{array}{l}\text { Proposed } \\
\text { Method }\end{array}$ \\
\hline $50 \%$ & $89 \%$ & $93 \%$ \\
\hline $70 \%$ & $92 \%$ & $96 \%$ \\
\hline $80 \%$ & $94 \%$ & $99 \%$ \\
\hline $100 \%$ & $96 \%$ & $98 \%$ \\
\hline
\end{tabular}

The proposed method of face recognition using Directional binary code is compared with the existing method of image identification using local binary pattern and showed the improved performance of the suggested method in the above mentioned Table.3.

Table 3 :Comparison between traditional and proposed face recognition System

\begin{tabular}{|c|c|c|}
\hline Alogorithm & $\begin{array}{c}\text { Recognition } \\
\text { Accuracy }\end{array}$ & $\begin{array}{c}\text { Computational } \\
\text { Time (s) }\end{array}$ \\
\hline $\begin{array}{c}\text { Traditional Local } \\
\text { Binary Pattern } \\
\text { basec Face } \\
\text { Recognition }\end{array}$ & $67.8 \%$ & 6385 \\
\hline $\begin{array}{c}\text { Proposed DBC } \\
\text { based Face } \\
\text { Recognition }\end{array}$ & $88.5 \%$ & 237 \\
\hline
\end{tabular}

The above table 3 gives the comparison among traditional and proposed face recognition system in terms of simulation parameters such as computational time and recognition accuracy.

\section{V.CONCLUSION}

The proposed methodology to increase the image recognition rate by retrieving the image from the database images. The extraction of the feature is done by Directional Binary Code and to create blurring various filters is used such as Gaussian filter, Average filter and Motion filter. We then include the Multi-SVM classifier to match with different images in the database. The introduced methods are analyzed and evaluated image recovery tests on ten databases which consists of images from natural scene and also of different color textures. The result is calculated by showing the matched images with input image.

\section{REFERENCES}

1. Dubey et al "Multichannel decoded LBP for content based image retrieval", IEEE Transaction of image processing, 2016

2. Abhijith et al "Face recognition across non uniform motion blur, illumination and pose" IEEE 2015.

3. Renliang et al "Robust point set matching for partial face recognition", IEEE 2016.

4. Shshbaz Majeed "Face recognition using fusion of LBP\& Zernike moments" IEEE conference 2016

5. [5] Shabat et al "Directional LBP for texture analysis" ICIAR 2016

6. Zhenhua Guo et al "Robust texture image representation by scale selective LBP" IEEE Transaction 2016.

7. Agnes Shifani, S \& Revathy, S \& Nanammal, V. "Dynamic facial tracking based on facial expressions using GLCM algorithm” Dusunen Adam. 10. 1308-1312, 2019

8. Jagadeesh et al "DBC based face recognition using DWT" Signal \& Image Processing: 2012.

9. G. Ramkumar, E. Logashanmugam, "Multimodal verge for scale and pose variant real time face tracking and recognition", IJEECS, 2019

10. Zhi Liu et al "robust blurred face recognition using sample-wise kernel estimation and random compressed multi scale LBP histogram" ICIP, 2014

11. Ying Zilu et al "Facial expression recognition based on NMF and SVM" IFIT, 2009.

12. Meng et al, "Finger vein recognition based on LDC" (2012).

13. Ramanathan et al "A support vector machine approach for efficient facial expression recognition" 2009 ICARTCC

14. Santhosh et al "Local directional mask maximum edge pattern for image retrieval and face recognition" IET 2015.

15. J.-P.Leduc, J.-M.Odobez C.Labit "Motion compensated adaptive wavelet filtering for image sequence processing" IEEE 1995.

16. Ramkumar $\mathrm{G}$ et al "Study on impulsive assessment of chronic pain correlated expressions in facial images" Biomedical Research, 2018

17. Mrtyunjay Ghosh, Srabani Mukhopadhyaua "Cache oblivious algorithm of average filtering in image processing" IEEE/OSA/IAPR International Conference on Informational, Electronics \& vision 2012.

18. Madronal et al "Hyper spectral image classification using a parallel implementation of the linear SVM on massively parallel processor array platform" IEEE 2016.

19. F. Maes et al "Multimodal image registration by maximization of mutual information," IEEE Trans. Med. Image, 1997.

20. Suri et al, "Mutual-information-based registration of TerraSAR-X and Ikonos imagery in urban areas," IEEE Trans. 2010.

21. Ghorbani et al "Multiresolution registration of multitemporal remote sensing images by optimization of mutual information using a simulated annealing-based Marquardt-Levenberg Technique," in Proc. Int. Conf. Intell. Adv. Syst., 2007.

22. Falco et al "Differential evolution as a viable tool for satellite image registration," Appl. Soft Comp., 2008. 
23. Suri et al "Combining mutual information and scale invariant feature transform for fast and robust multisensor SAR image registration," 75th ASRPS Conf., USA, 2009.

24. Heo et al, "Mutual information-based stereo matching combined with SIFT descriptor in log-chromaticity color space," in Proc. CVPR, 2009.

\section{AUTHORS PROFILE}

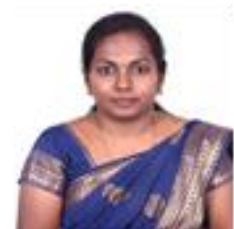

M.Tamilselvi is Research scholar from Dept of ECE at Sathyabama Institute of science and technology, Chennai, India. She completed her UG in ECE in the year 2005 from C.Abdul Hakkem College of Engineering and Technology, Vellore and received her M.Tech in VLSI Design from Sathyabama University in 2013. She is having more than 10 years of teaching experience in various engineering colleges and published many research papers in National and International journals and conferences.

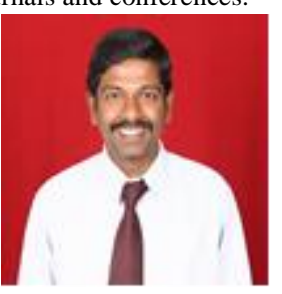

Dr.S.KARTHIKEYAN was born in 1979. He received his Bachelor's degree in Electronics and Instrumentation Engineering from Annamalai University in 2002. He received a Master's degree in Applied Electronics from Sathyabama University, Chennai in 2007. He received Ph.D in Wireless sensor Network from Sathyabama University in 2014. He is currently working

as an Associate Professor in the Department of Electronics and Communication Engineering, besides coordinator, International Affairs school of electrical and electronics engineering. Sathyabama Institute of science and technology, Chennai. His areas of interest include Mobile Agent, Wireless Sensor Networks, Wireless Communication, Image Processing, Signal Processing, IoT and Cryptography. He has about 17 years of experience in teaching. Board of studies Member in Vel's university, Chennai. He has published 45 papers in International / National Journals/ conferences. He was a reviewer in a top Journals / conferences like Elsevier, Inderscience, etc., He guided 20 PG students in India and a PG student in Rostock university, Germany. Doctoral committee member in Anna university, Vel's institute and Veltech institute. Nodal In charge for CDAC PG courses in Sathyabama. Active involvement in MOU with industrial 